\title{
Glomerulonefritis primarias en niños que asisten a un Centro de Referencia en la Región Caribe colombiana
}

\author{
Gustavo Aroca Martínez ${ }^{1-3}$, Ariel Polo Castillo ${ }^{1-3}$, Andrés Cadena Bonfantti ${ }^{1,2}$, \\ Henry J. González Torres ${ }^{2}$, Hernando Padilla Galindo ${ }^{3}$, Santos Ángel Depine ${ }^{2,4}$ \\ $1_{\text {Clínica de la Costa Ltda. (Baq, Atl) }}$ \\ 2 Programa de Medicina, Universidad Simón Bolívar (Baq, Atl, Col) \\ 3 Facultad de Medicina, Universidad del Norte (Baq, Atl, Col) \\ ${ }^{4}$ Confederación de Asociaciones de Diálisis de la República Argentina (BA, BA, Arg)
}

\section{Resumen}

Introducción: Las glomerulonefritis (GN) primarias son enfermedades que afectan a la estructura y función del glomérulo. Dentro de las glomerulopatías proliferativas se ha observado que la GN mesangial por IgA e IgM, son las que evolucionan con mayor frecuencia a la insuficiencia renal permanente; seguida de la GN membrano-proliferativa o mesangiocapilar. La presentación clínica de la GN incluye hematuria, proteinuria e insuficiencia renal aguda o crónica.

Material y métodos: Se revisaron las historias clínicas y se acopiaron en una base de datos todas las biopsias realizadas entre los años 2008 a 2014. Se realizó un $\chi^{2}$, para establecer las asociaciones entre variables $(\alpha$ $=95 \%$ ) y para las diferencias entre proporciones se utilizó t de Studentó U- Mann-Whitney.

Resultados: Se seleccionaron 146 pacientes ( $88 \%$ del total analizado) que tenían los datos completos. La edad promedio fue de $8 \pm 4$ años para ambos sexos. No se encontraron diferencias significativasni asociaciones entre el sexo y la edad ( $\mathrm{p} \geq 0,05)$. Las GN predominantes fueron las proliferativa mesangial y la nefropatía por IgA. Las GN por membrana basal delgada, nefropatía por $\mathrm{C} 3$ y $\mathrm{C} 4 \mathrm{q}$ no se presentaron en varones. La presentación clínica más frecuente fue el Síndrome Nefrótico (58\%).

Conclusiones: Los hallazgos de éste registro de biopsias renales podrían ser la base para la creación de un Registro de Glomerulopatías en niños en Colombia, instrumento necesario para establecer programas de tratamiento y prevención de las enfermedades glomerulares en nuestro país a fin de disminuir su progresión.

Palabras clave: glomerulonefritis, niños, proteinuria, hematuria.

Prymary glomerulonephritis in children in a medical center of Colombian caribbean

\section{Summary}

Introduction: primary glomerulonephritis are diseases that affect the structure and function of the glomerulus. Proliferative glomerulopathies for it has been observed that for GN mesangial IgA and IgM, which are mostly evolve into chronic renal insufficiency; followed by membrane proliferative GN or mesangioca- 
pilar. The clinical presentation of the GN is the result of the combination of hematuria, proteinuria, and the presence of chronic or acute renal failure.

Material and methods: We reviewed the medical records. They were collected in a database of all biopsies performed from 2008 to 2014 one chi-square was conducted to establish the associations between variables $(\alpha=95 \%)$. Differences between proportions was used T-Student or U-Mann - Whitney.

Results: 146 (88 \%) patients who had complete data were selected. The average age was $8 \pm 4$ years for both sexes. No significant difference between gender by age ( $p \geq 0.05)$, nor an association between gender and age ( $\mathrm{p} \geq 0.05)$. The most predominant GN Mesangial Proliferative and were IgA nephropathy. The GN Thin Basement Membrane, nephropathy $\mathrm{C} 3$ and $\mathrm{C} 4$ patients showed no men. The syndromic box was more prevalent Nephrotic Syndrome (58\%).

Conclusions: The existence of this record renal biopsy is the basis for the creation of the register of glomerulopathies in children in Colombia, whose data are needed to establish treatment programs and prevention of glomerular diseases in our country to reduce its progression.

Key words: Glomerulonephritis, children, proteinuria, hematuria.

\section{Introducción}

$\mathrm{G}$ lomerulonefritis primaria hace referencia a enfermedades que afectan a la estructura y función del glomérulo, aunque después se pueden ver implicados los demás componentes de las nefronas. Su etiología es desconocida la mayoría de las veces y no suelen ser secundariasa procesos sistémicos conocidos, quedando confinadas al riñón ${ }^{1}$. La gran mayoría son de base inmunológica, desconociéndose el proceso desencadenante, lo que la convierte en un factor muy importante en la génesis de la enfermedad. Por otra parte, los factores genéticos juegan un rol condicionante en la vulnerabilidad individual.

Por su etiología, las glomerulopatías se dividen en proliferativas (endotelio capilar, mesangio proliferativa, membrano proliferativa y proliferación epitelial) y no proliferativas (cambios mínimos, esclerosis focal y segmentaria, membranosa y mesangial leve $)^{1,2}$.

En las glomerulopatías proliferativas la observación epidemiológica permite inferir que en la GNmesangial por IgA e IgM, el $15-40 \%$ de los niños evolucionarán a una insuficiencia renal crónica (IRC), mientras que en la GN membrano-proliferativa o mesangiocapilar, este porcentaje se acercará al 80\% de los casos. La Glomerulonefritis endocapilar difusa esla más frecuente en la edad pediátrica, y el SíndromeNefrítico Agudo (SNA)es su forma habi- tual de presentación clínica, generalmente con buen pronóstico. Contrario a ello, tanto en frecuencia como en pronóstico, se encuentra la Glomerulonefritis extracapilar, también conocida como glomerulonefritis rápidamente progresiva, con un $75 \%$ de mortalidad y/o necesidad de diálisis a los 2 años de evolución ${ }^{3-5}$.

La presentación clínica de las glomerulopatías habitualmente incluye a la hematuria (microscópica o macroscópica), proteinuria (de rango nefrótico o no) e incluso la insuficiencia renal aguda o crónica. Se puede asociar hipertensión arterial. En las glomerulopatías no proliferativas, la GN de cambios mínimoses la causa más frecuente de síndrome nefrótico en niños, sin embargo su pronóstico es bueno en la mayoría de los pacientes. La GN segmentaria y focal es el segundo tipo más frecuente observado en el síndrome nefrótico y el 50\% evoluciona a una insuficiencia renal crónica (IRC), mientras que la GN membranosa, es inusual en niños y representa menos del $10 \%$ del todas las glomerulopatias primarias y no se acompaña de insuficiencia renal ${ }^{3,4}$.

En un resumen, se puede concluir que en su presentación clínica predominarán las alteraciones urinarias asintomáticas, incluyendo hematuria (macro y microscópica) o síndrome nefrótico y/onefrítico. El objetivo de la presente investigación fue caracterizar clínica y epidemiológicamente las GN primarias diagnosticadas en niños que fueron sometidos a biopsia renal en un centro de referencia del Caribe Colombiano. 


\section{Materiales y métodos}

La información se recolectó de las historias clínicas acopiadas en la plataforma NefroRed $\AA$, previa autorización del Comité de Ética del Centro de Referencia de la Red. Se incluyeron todos los pacientes hasta la edad de 17 años, a quienes; entre los años 2008 y 2014, se les había realizado una biopsia renal como consecuencia a haber presentado un cuadro clínico compatible con una GN primaria para su confirmación por el Departamento de Nefropatología.

El estudio fue descriptivo, transversal, la información fue recogida en la ciudad de Barranquilla, Colombia, que es la ubicación geográfica del centro de referencia que realizó las biopsias y analizó las muestras de los pacientes de toda la región Caribe Colombiana.

Para esta investigación, se incorporó a una base diseñada específicamente, toda la información clínica y anatomo patológica de los pacientes a quienes se les había efectuado una biopsia renal entre los años 2008 a 2014. Las variables analizadas para este trabajo incluyeron los datos de procedencia de los pacientes, su edad, sexo, el tiempo de evolución y el cuadro clínico. Los análisis estadísticos incluyeron la medición de la tendencia central y prueba de $\chi^{2}$ para establecer eventuales asociaciones entre variables $(\alpha=95 \%)$ y para establecer posibles diferencias entre proporciones se utilizó el t de Student o U- Mann-Whitney- Los datos fueron analizados con R6.

\section{Resultados}

En total se revisaron 165 historias clínicas, de las cuales se seleccionaron 146 pacientes $(88 \%$ de la población) dado que solo estos tenían completo el diagnostico histológico, la edad, el sexo y la procedencia. Igualmente, solo se tuvieron en cuenta los pacientes que tenían residencia en la Región Caribe colombiana.

La distribución por sexo fue de tres (3) mujeres por cada cuatro(4) hombres. El sexo masculino tuvo una mayor prevalencia que el femenino $(\mathrm{t}=2,4 ; \mathrm{p}$-valor $<0,05 ; \alpha=95 \%$ ).

Analizando la distribución de los pacientes, fue posible observar que el 64\% de los mismos, fueron del
Figura 1.

Distribución por sexo. Fuente: Datos del estudio.

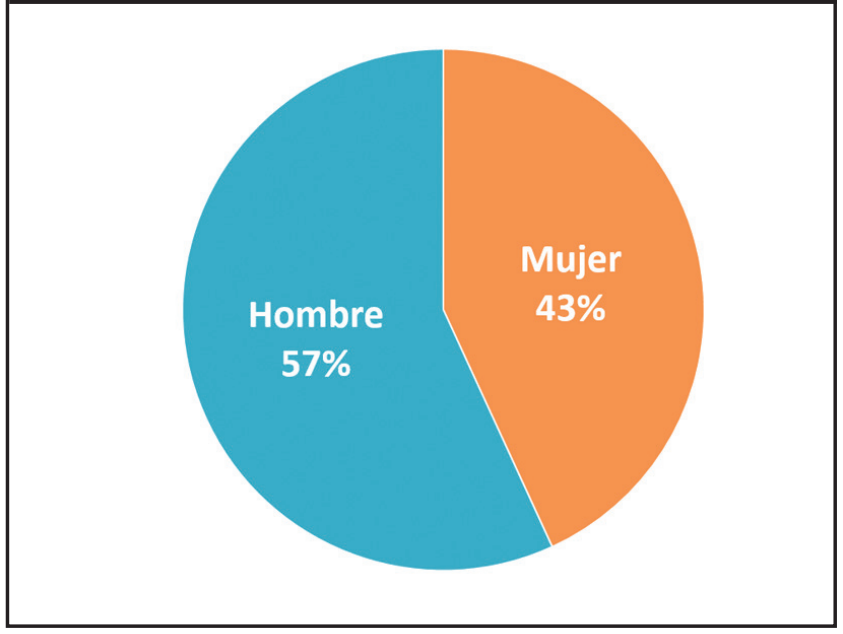

mismo Departamento, lo cual podría explicarse por la ubicación geográfica del centro de referencia. Sin embargo, debe destacarse que se controlaron pacientes de toda la Región Caribe colombiana, incluyendo a los provenientes de la Región Insular, en este caso San Andrés y Providencia, con una representación del $1 \%$.

Se estudiaron 83 niños y 63 niñas, la edad promedio fue de $8 \pm 4$ años para ambos sexos, la edad máxima reportada 17 años cumplidos. Al analizar la relación sexo con edad no se encontraron diferencias significativas entre los sexos $(\mathrm{t}=0,31 ; \mathrm{p}$-valor $\geq 0,05 ; \alpha=$ $95 \%$ ), así como tampoco se encontró una asociación entre el sexo y la edad $\left(\chi^{2}=2,21 ; p\right.$-valor $\geq 0,05 ; \alpha$ $=95 \%$ ).

Las GN predominantes fueron las GN proliferativa mesangial y Nefropatía por IgA. El 50,79\% de las niñas y el 55,42 \% de los niños estudiados presentaron una GN proliferativa mesangial, y la Nefropatía por IgA afectó al $22 \%$ de las niñas y al $21 \%$ de los niños incluidos en el estudio.

Las GN por membrana basal delgada y la Nefropatía por $\mathrm{C} 3$ y $\mathrm{C} 4$ no se presentaron en niños de sexo masculino, mientras que la GN proliferativa mesangial y la Nefropatía por IgM, se presentaron en una mayor proporción en ese sexo, a pesar de lo cual al comparar las proporciones entre sexos de cada una 
Figura 2.

Distribución por Departamento de procedencia. Fuente: Datos del estudio.

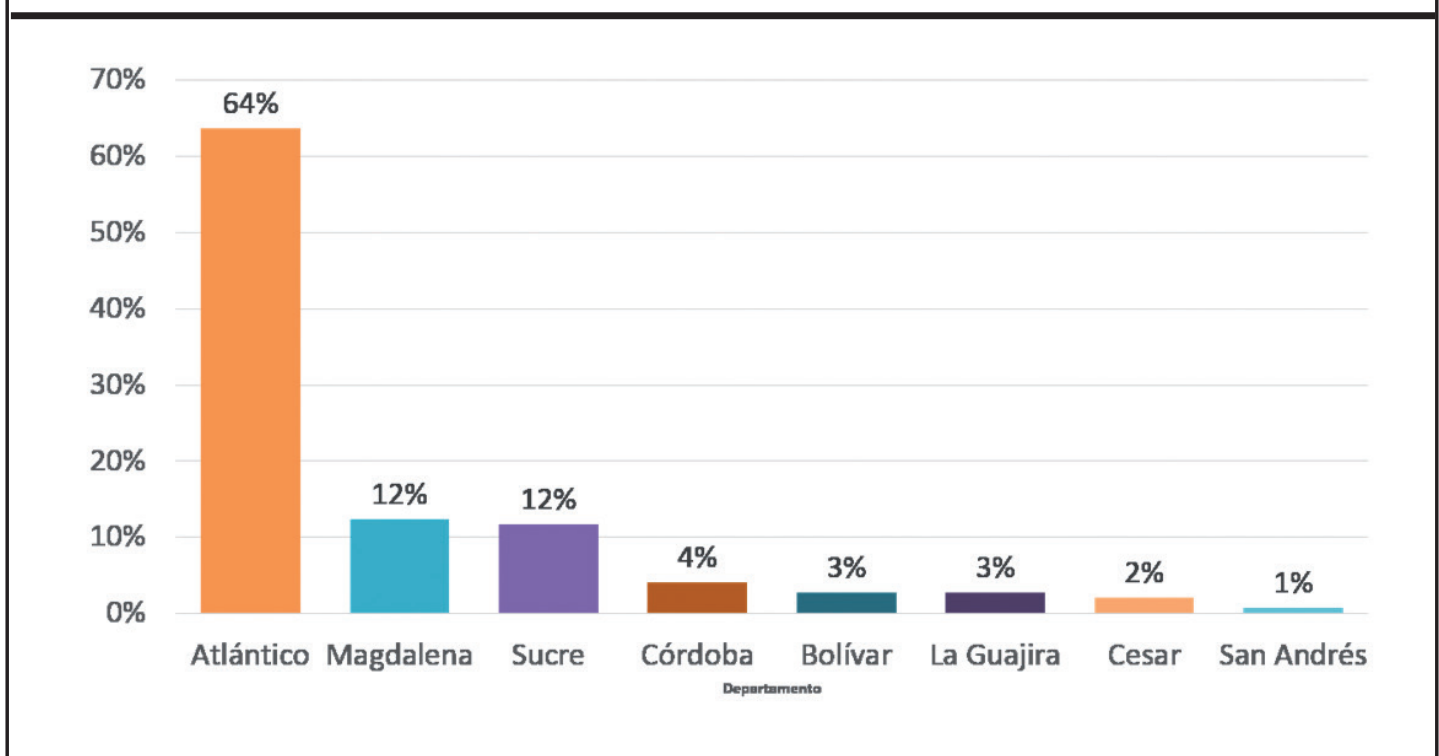

Figura 3.

Distribución de los pacientes por Etapa de Desarrollo. Fuente: Datos del estudio.

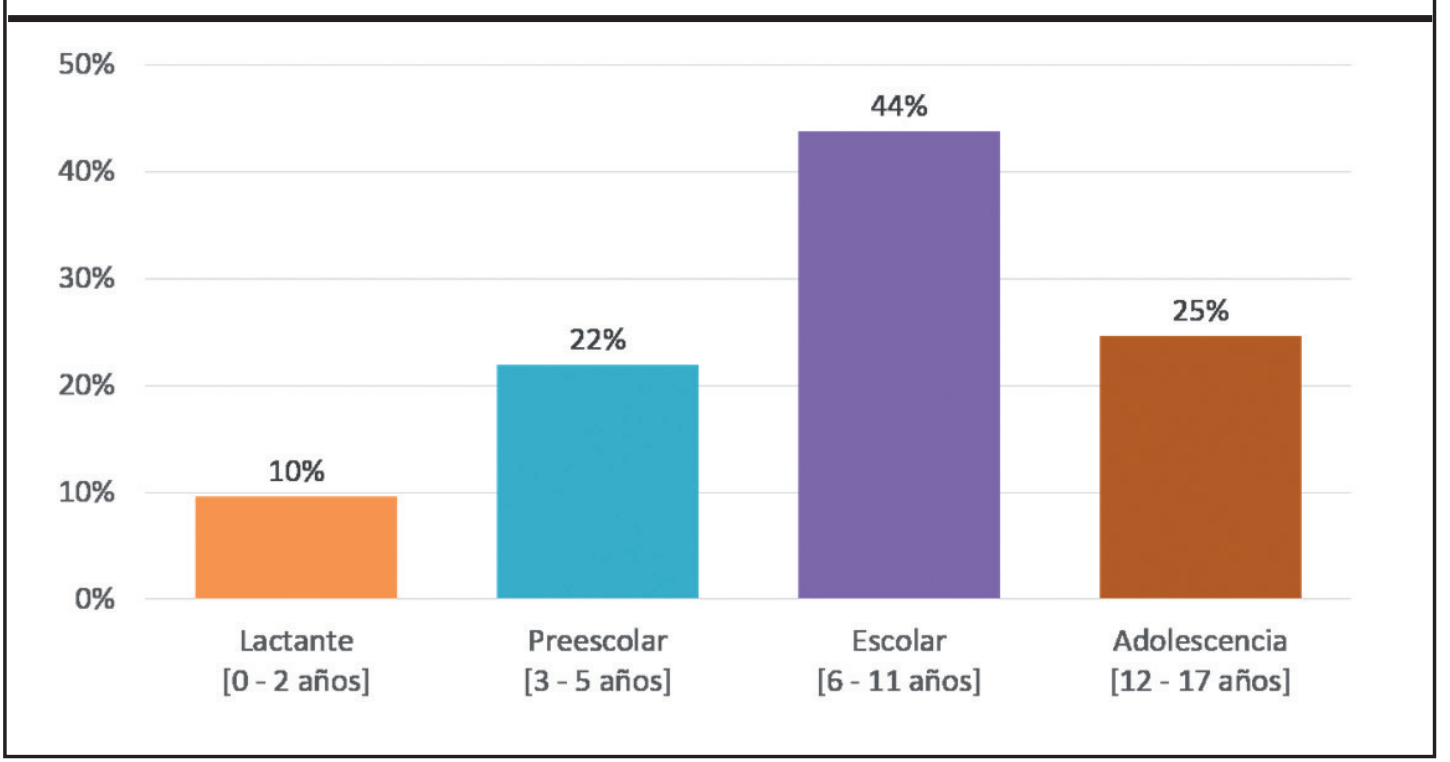

de las GN no se encontraron diferencias significativas $(\mathrm{W}=-6,0 ; \mathrm{p}$-valor $\geq 0,05 ; \alpha=95 \%)$. Tampoco se pudo demostrar una asociación entre el sexoy la GN $\left(\chi^{2}=8,53 ; p\right.$-valor $\left.\geq 0,05 ; \alpha=95 \%\right)$.
El cuadro sindrómico más habitual fue el Síndrome Nefrótico (58\%), seguido de Hematuria (35\%). El 59,04\% (49 de 83) de las niñas y el 57,14\% (24 de 63) de los niños presentaron Síndrome Nefrótico, 
Figura 4.

Distribución de las GN Fuente: Datos del estudio.

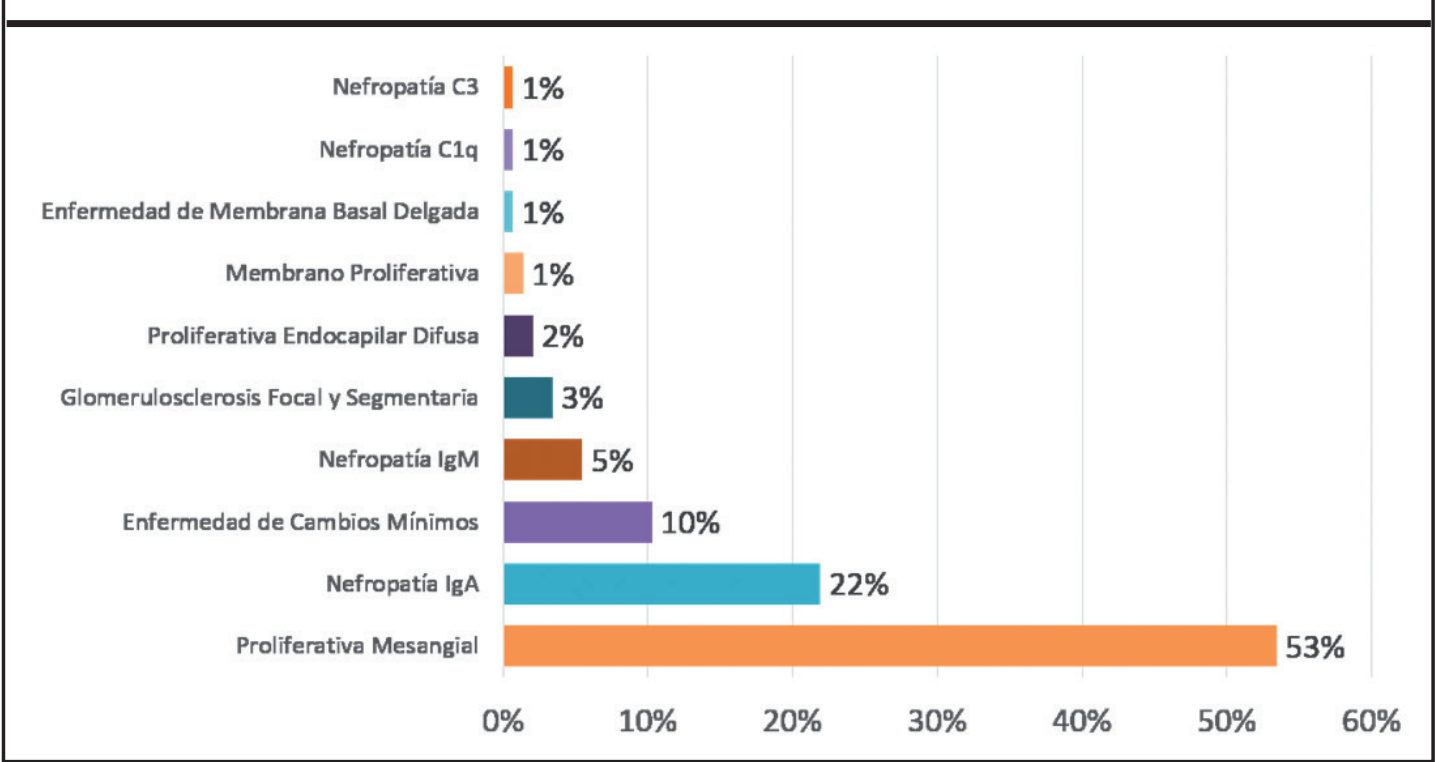

Figura 5.

Distribución según cuadro sindrómico. Fuente: Datos del estudio.

jindrome Nefrítico

$3 \%$

Proteinuria

$3 \%$

Hematuria

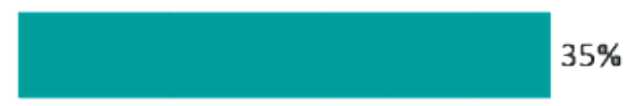

indrome Nefrótico

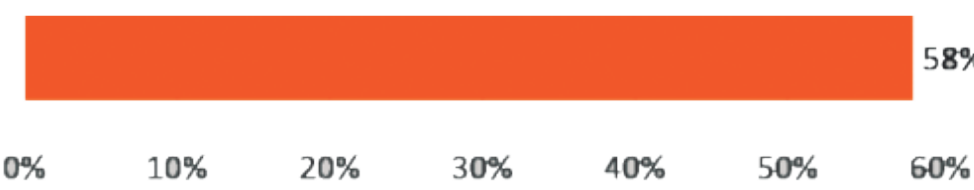

$58 \%$

$0 \%$

$10 \%$

$40 \%$

$60 \%$

709

mientras que la Hematuria se manifestó en el 32,4\% (27 de 83) de las niñas y el 38,1\% (24 de 63) de los niños, incluidos en el estudio.

El Síndrome Nefrítico y la insuficiencia renal sólo se presentaron en pacientes de sexo masculino, mien- tras que la hematuria y la proteinuria, se presentaron en una mayor proporción para el sexo femenino, pero sin diferencias estadísticamente significativas $(\mathrm{t}$ $=0,0 ; p$-valor $\geq 0,05 ; \alpha=95 \%$ ). De la misma forma, al investigar una posible asociación entre el sexo y el 
cuadro sindrómico, los resultados fueron estadísticamente no significativos $\left(\chi^{2}=4,173 ; p\right.$-valor $\geq 0,05 ; \alpha$ $=95 \%$ ).

\section{Discusión}

El síndrome nefrótico primario (SNP) es la manifestación clínicamásfrecuente de las Glomerulopatías primarias en la infancia. Siendo esta a su vez una causa frecuente de consulta al nefrólogo pediatra.

Generalmente afecta a niños de 1 a 6 años de edad. La incidencia anual de la enfermedad varía entre 2 y 7 casos nuevos en menores de 16 años ${ }^{7,8}$.

Según datos del Grupo de Estudio Colaborativo Internacional sobre Enfermedades Renales Pediátricas $(\mathrm{GECI})^{9}$, el 78\% de los niños con síndrome nefrótico es corticosensible y en un 91,8\% la histología demostró una lesión de cambios mínimos en la biopsia renal.

En una serie de 134 niños con SNP y biopsia renal con lesiones glomerulares mínimas, Gordillo et al ${ }^{10}$ encontraron su presencia con una distribución etaria entre los 9 meses y los 10 años de edad. La proporción de casos del sexo masculino al femenino es $2: 1$, igualándose en adolescentes y adultos. En GECI la distribución fue semejante ${ }^{10,11}$.

En nuestro estudio encontramos una mayor prevalencia de SNPen el sexo masculino, similar a lo descrito por la literatura, al igual que los grupos etarios de presentación, siendo menos frecuente en mayores de 15 años.

Con respecto a la Nefropatía por IgA, su frecuencia geográfica varía en diferentes regiones del mundo. Se observa en más del $40 \%$ de las biopsias realizadas por enfermedad glomerular en Asia, comparada con el $20 \%$ en Europa y el $10 \%$ en Norteamérica. En Asia, es habitual realizar análisis a escolares y la biopsia renal se hace en pacientes con hematuria asintomática, lo cual permite una detección precoz de la enfermedad, lo que justificaría su mayor prevalencia $^{12,13}$. En nuestro estudio se encontró Nefropatía por IgA con menor frecuencia de lo reportado, al igual que la Nefropatía con cambios mínimos, aunque corresponde manifestar que existen diferentes criterios en los distintos centros que aportan sus da- tos a Nefrored para indicar la realización de biopsia renal, lo cual podría generar un sesgo en los resultados, pudiendo poner en evidencia un diagnóstico tardío o un subregistro de la enfermedad.

Lo reportado con respecto a los hallazgos histopatológicos en los cuales la proliferación mesangial fue la más frecuente pero con una diferencia estadísticamente significativa a lo reportado por otras series, tales como Bolaños et al sobre 73 biopsias reportadas en Cali, Colombia en la que se encontró proliferación mesangial en un $46 \%{ }^{14}$, la de Gordillo et al en México, sobre 213 biopsias que mostró la misma patente histológica en un $6 \%$, Gulati et ${ }^{15}$ al en una comunicación sobre 222 biopsias en la India, la encontraron en un $8 \%{ }^{16}$, permitiría inferir la existencia de algún otro factor, ya sea genético, étnico, ambiental o nutricional que permita explicar las variaciones de las distintas formas de manifestación histológica de las glomerulopatias en diferentes lugares del mundo, lo cual amerita la realización de otras investigaciones para analizar estas variables de manera independiente.

En cuanto a la glomerulonefritis con proliferación endocapilar difusa, dada su buena evolución,con resolución espontánea y progresiva de todo el cuadro clínico, determina que escasamente un 2\%17, llegue a la biopsia renal cuando se sospecha la posibilidad de secuelas a largo plazo, en nuestro estudio, su frecuencia resultó similar a las encontradas en otras series.

Como un aporte adicional a esta investigación, nuestro grupo infiere que la concreción de este registro de biopsias renales y sus hallazgos, generan una fuerte posibilidad de ampliarlo, dando origen a la creación del Registro de Glomerulopatías en niños en Colombia, cuya importancia es la de generar una base de datos nacionales, que permita establecer programas de tratamiento y prevención de las enfermedades glomerulares en nuestro país a fin de disminuir su progresión al estadio permanente.

\section{Agradecimientos}

A la doctora Zilac Espitaleta V. Nefróloga Pediatra Hospital Universitario San Ignacio por los aportes y correcciones de este artículo. 


\section{Conflicto de interés}

Los autores declaran que no tienen conflicto de interés.

\section{Bibliografía}

1. Ortiz ER. Síndrome nefrótico pediátrico. In: Asociación Española de Nefrología Pediátrica, editor. Protocolos de Nefrología. Asociación Española de Pediatría; 2014. p. 283-301.

2. Pietrángelo C. Las Glomerulopatías. Enfoque Clínico-patológico. Rev med interna2000;2(4). Available from: http:// www.smiba.org.ar/med_interna/vol_02/03_05.htm

3. Fernández Fresnedo G. Glomerulonefritis primarias. Nefrología al día. p. 23-45.

4. Piña J, Saieh C. Hematuria en pediatría. RevMedClin Condes. 2009;20(6):904-10.

5. Luque A, Reyes A, Canal MJ, Gómez-Campdera FJ, Morales MD. Causas y progresión de la insuficiencia renal crónica en la infancia. Nefrología. 1988;8(3):265-72.

6. R Core Team. R: A Language and Environment for Statistical Computing [Internet]. Vienna, Austria: R Foundation for Statistical Computing; 2014. AresesTrapote R, Sanahuja Ibáñez MJ, Navarro M. [Epidemiology of chronic kidney disease in Spanish pediatric population. REPIR II Project]. Nefrologia 2010 Jan [cited 2014 Dec 7];30(5):508-17.

7. Eddy A, Symons J. Nephrotic syndrome in childhood. Lancet 2003; 362:629-39.

8. Schlesinger E, Sultz H, Mosher W, Feldman J. The nephrotic syndrome: its incidence and implications for the community. Am J Dis Child 1968; 116:623-24.

9. Gofrey A, Barrat M. Responsive nephrotic syndrome. En: Barrat T, AdverE,HarmonW. Pediatricneprhology 7.Ed. Baltimore : Lipppincott Williams \&Wilkins; 2007, p 731-47.

10. Ingulli E,Tejani A. Racial differences in the incidence and renal outcome of idiopathic focal segmental glomerulosclerosis in children. PediatrNephrol. 1991;5:393-7

11. Koch V, Gordillo G. Sindrome Nefrótico. En : Gordillo G, Exeni R, De La Cruz J Editores. NefrologiaPediatrica 3 Ed. Elsevier España; 2009 p 284-302

12. CattranDC,CoppoR.Cook HT, et al: The Oxfordclassification of IgAnephropathy :rationale,clinicopathological correlations, and classification. Kidney International .2009; 76 (5) : 534-45

13. Coppo R. Pediatric IgA nephropathy:clinical and therapeutic perspectives. Seminars in Nephrology. 2008 ; 1: 18-26.

14. Bolaños L,Castaño I. Características Clínicas e histopatológicas del sindrome nefrótico primario. ColombMéd 2005 , 36; $1: 29-33$

15. Gordillo PG,Garcia A, Mota HF. Síndrome nefrótico Ideopático. Rev. Mexicana Pediatria 1970, 39:121-126

16. Gulati S, Sharma AP, SharmaRK. Do current recommendations for kidney biopsy in nephrotic syndrome need modifications? PediatrNeprhol 2002; 17:404-08.

17. Mehls O, Rigden S, Ehrich JHH, Berthoux F, Jones EHP, Valderrabano F, on behalf of the EDTA-ERA Registry. Report on management of renal failure in Europe, XXV, 1994. The child-adult interface. Nephrol Dial Transplant 1996:11(Suppl 1):22-36 